\title{
Safety and Efficacy of Coil Embolization in Treating Vascular Complications of Pancreatitis
}

\author{
${ }^{1}$ Dr. Prajwaleet Gour, ${ }^{2}$ Dr. Jawahar Rathod, ${ }^{3}$ Dr. Dharmik Bhuva, \\ ${ }^{4}$ Dr. Dyaneshwar Shinde, ${ }^{5}$ Dr. Ashwini Bakade, \\ ${ }^{1,2,}$ Associate Professor, ${ }^{3}$ Fellow, Interventional Radiology, ${ }^{4}$ Junior Resident, ${ }^{5}$ Assistant Professor, \\ *Department of Radiology, Government Medical College, Nagpur
}

\begin{abstract}
:
Introduction: Acute pancreatitis is an acute inflammatory process of the pancreas. Most patients recover without any major sequelae; however a minority of patients have life threatening vascular complications. Vascular complications of pancreatitis include arterial erosion, pseudo aneurysm, ischemic complications, remote vascular complications, venous thrombosis and varices. We studied patients to study safety and efficacy of coil embolization in treating vascular complications pancreatitis and to reduce morbidity and mortality rates. Methods: This prospective study was conducted from July 2014 to February 2016 at Department of Radiology, Government Medical College Nagpur, in which all patients who presented with vascular complications of pancreatitis were enrolled to undergo embolization using coiling, $N$-butyl cyanoacrylateglue or gluefoam. The patients were followed to see rebleeding and treatment failure rates.

Results: 38 patients were included in the study, of which 33 had pseudoaneurysm and 5 had arterial wall erosions. The most common artery involved was splenic artery in both acute and chronic pancreatitis patients. For treatment, only embolization coils were used in $76 \%$ of the patients. There were no occurrences of bowel ischemia, hepatic insufficiency, or rupture-related deaths in any of patients in the follow-up period.

Conclusions: Results of embolization of pseudoaneurysms with coils showed high success rate and good short \& long term outcome. More research is required to search for better embolization materials which can minimize the risk of rebleeds or other complications.
\end{abstract}

Keywords: pancreatitis, endovascular, coiling, embolization, treatment, safety

\section{Introduction}

Acute pancreatitis is an acute inflammatory process of the pancreas. Acute pancreatitis should be suspected in patients with severe acute upper abdominal pain but requires biochemical or radiologic evidence to establish the diagnosis. Most patients with acute pancreatitis have acute onset of persistent, severe epigastric abdominal pain. In some patients, the pain may be in the right upper quadrant or, rarely, confined to the left side.Most patients recover without local or systemic complications or organ failure and do not have recurrent attacks. However, a small proportion of patients with acute pancreatitis have necrosis of the pancreas or peripancreatic tissue and complications due to pancreatitis. These patients have a high overall mortality. Local complications of acute pancreatitis include acute peripancreatic fluid collection, pancreatic pseudocyst, acute necrotic collection, and walled-off necrosis.In the literature, major vascular complications of pancreatitis occur with a frequency of $1.2-14 \%$, with a greater incidence seen in chronic pancreatitis (7-10\%) than acute pancreatitis $(1-6 \%)$ and it's related to severity of disease. Hemorrhage is usually due to erosion of a major pancreatic or peripancreatic blood vessel or due to the formation and subsequent rupture of an arterial pseudoaneurysm.

Vascular complications of pancreatitis include arterial erosion, pseudo aneurysm, arterial / micro vascular ischemic complications(local), remote vascular complications (fat emboli, avascular necrosis), venous thrombosis and varices. Angiographic control of gastrointestinal (GI) vascular complications is indicated for patients with upper or lower GI bleeding who fail to respond to medical and/or endoscopic therapy. We studied patients to study safety and efficacy of coil embolization in treating vascular complications pancreatitis and to reduce morbidity and mortality rates.

\section{Study design and patient population}

\section{Methodology}

After taking approval from the institutional ethics committee we enrolled the patients from the inpatient ward. All consecutive patients who were admitted to the surgical and medical ward of Government Medical College, Nagpur for bleeding as a consequence of pancreatitis were enrolled for the study. The decision to perform coil embolization was taken by the consultant based on patients' presentation and previous medical 
history. We designed a prospective study where patients with GI bleed due pancreatic/ peripancreatic pseudo aneurysm \& arterial erosions presented with malena or hematemesis and pain. We excluded patients with upper GI bleeds due to varices or other reasons. All the patients included in the study underwent standard preoperative investigations, including rdiographic imaging. Study's primary end points was the technically defined as successful exclusion of pseudoaneurysm from systemic circulation, confirmed by Digital Subtraction Angiography at end of procedure.

The basic instruments which were used for coiling were single wall puncture needle, 5 french angiosheath, 4 french cobra catheter $/ 4$ french SIM 1 catheter, 0.035 inch angled guide wire, 2.7 french micro catheter, $370 \mathrm{mg} / \mathrm{ml}$ iodinated contrast, $3-5 \mathrm{~mm}$ metallic embolization coils, N-butyl cyanoacrylate(NBCA) embolising glue/gelfoam. The coils were made of either stainless steel or platinum. Polyester fibers are incorporated into the body of the coil to increase its thrombogenicity.

\section{Study setting}

The study was performed in the Department of Radiology, Government Medical College Nagpur. Nagpur is the he third largest city of the Indian state of Maharashtra with the metropolis population of 2,405,665 according to 2011 census. Government Medical College Nagpur is a 1400 bedded tertiary level hospital located in south Nagpur.

\section{Data collection and analysis}

We enrolled and collected data from July 2014 to February 2016 and then analysis of the records was carried out. Data obtained from hospital was codified and entered into Microsoft excel sheets. Data were then imported in to Statistical Package for Social Sciences (SPSS) version 21 and descriptive analysis was performed using appropriate statistical analysis. Follow-up imaging was done with either a Doppler study and/or a contrast enhanced computed tomography. The post procedure mean time of follow-up was four months (range 7 days -48 months).

\section{Results}

A total of 38 patients satisified our inclusion and exclusion criteria during the stipulated period of the study. Out of 38 patients 32 were male (84.2\%) and 6 were female (15.8\%). The age of the patients ranged from 18 years to 54 years. The most common age group was 30 to 39 years. (Table 1)In 38 patients of arterial complication total $33(86.9 \%)$ patiens had pseudoaneurysm and $5(13.1 \%)$ patients had arterial wall erosion with direct extravasation of contrast material. Of all the patiens enrolled in the study, 10 were diagnosed with acute pancreatitis and 28 had chronic pancreatitis. The most common artery involved with pseudoaneurysm in patients with acute pancreatitis was splenic artery (40\%). Similarly splenic artery was the most common artery involved $(39 \%)$ in pseudoaneurysm in patients with chronic pancreatitis, followed by gastroduodenal artery (32\%). (Table 2) Only embolization coil was used in $76 \%$ of the patients, followed by coil and glue in $18 \%$ of the patients. (Table 3) The followup was available for 30 of 38 patients (79.9\%).One patient died 15 days after the endovascular procedure. The cause of death was sepsis related to pancreatitis. There were no occurrences of bowel ischemia, hepatic insufficiency, or rupture-related deaths in any of patients in the follow-up period.Average hemoglobin level before procedure were $6.0 \mathrm{mg} / \mathrm{dl}(4-8 \mathrm{mg} / \mathrm{dl})$, after 15 days post coiling follow up hemoglobin increased to average $9.6 \mathrm{mg} / \mathrm{dl}$.Procedure-related complications occurred in two patients. One of coils prolapsed in the proper hepatic artery. However, the hepatic artery remained patent. In another patient with splenic artery pseudoaneurysm treated with intra-arterial NBCA injection, NBCA spilled into distal splenic artery branches leading to a large splenic infarct.

\section{Discussion}

In the overwhelming majority of patients with upper or lower gastrointestinal (GI) bleeding, the bleeding either resolves spontaneously or can be controlled endoscopically. However, some patients have persistent or recurrent bleeding. Persistent or recurrent bleeding occurs in 7 to $16 \%$ of patients with upper GI bleeding and in up to $25 \%$ of patients with lower GI bleeding. Such patients may require angiographic intervention to locate and/or to treat the source of bleeding. Angiography provides the most detailed evaluation of vascular involvement and may diagnose the site and source of bleeding, indicated by erosive arterial changes or pseudoaneurysm formation. In most cases, angiographic control of gastrointestinal (GI) bleeding requires that the site of bleeding first be identified. This can be done with endoscopy, nuclear scintigraphy (tagged red blood cell scan), computed tomographic angiography, or standard angiography.Contrast extravasation into the bowel lumen is considered definitive evidence of a bleeding site. Indirect evidence includes visualization of an aneurysm or pseudoaneurysm, filling of spaces outside the bowel lumen (diverticula), early draining vessels (angiodysplasia), neovascularity (tumors), arteriovenous fistulas, and hyperemia (colitis).

Embolization works by mechanically occluding the arterial blood supply to the bleeding site. The goal of embolization is to decrease the blood flow to the bleeding site enough to achieve hemostasis while 
maintaining collateral perfusion to adjacent tissues to prevent ischemia or infarction. Microcoils are the preferred embolic agent option for treating GI bleeding by most interventionalists. Selective catheterization and embolization of distal arteries in the mesenteric circulation became possible with the introduction of microcatheters. Microcoils can be deployed selectively into the distal bleeding artery and can preserve the collateral blood supply to the area. Liquid agents have also been used for embolization. These agents include $\mathrm{N}$ butyl 2-cyanoacrylate (NBCA) and ethylene-vinyl alcohol copolymer. Once delivered, these agents solidify, leading to embolization. A potential advantage of NBCA is that the time required for embolization is shorter than that with other agents. However, its use requires significant training and has been associated with bowel ischemia and non-targeted embolization. Microcoils have become the preferred agent for embolizing bleeding vessels and are simply deployed via a microcatheter to the site of bleeding.

When technically feasible, embolization is initially successful in treating approximately $95 \%$ of patients with GI bleeding. However, as is seen with intra-arterial vasopressin, rebleeding is common (9 to $56 \%$, depending upon the bleeding site. In a review of 15 studies with 819 patients with upper GI bleeding, endovascular embolization with coils, polyvinyl alcohol particles, blood clots, gelatin sponge, or N-butyl 2cyanoacrylate (NBCA) was technically successful in 762 patients $(93 \%)$. Of those who underwent technically successful embolization, $549(67 \%)$ responded well clinically with cessation of bleeding. Rebleeding occurred in $33 \%$, though half responded to repeat embolization.

Factors associated with failed embolization for upper GI bleeding include multiorgan failure, anticoagulant use, corticosteroid use, pre-embolization vasopressor use, bleeding subsequent to trauma or invasive procedures, longer time to angiography, previous surgery, and embolization using coils alone or coils with another embolic agent. The complications of embolization include those associated with arteriography itself (eg, hematomas, arterial thrombosis, dissection, embolism, and pseudoaneurysm formation), and bowel infarction. In a systematic review that included 927 patients undergoing embolization for upper GI bleeding, complications occurred in 5 to $9 \%$ of patients, depending upon the type of bleeding (luminal or transpapillary), with ischemia and infarction accounting for the majority of complications.

\section{Conclusion}

Endovascular coil embolization is safe and effective procedure in treating and preventing vascular complications of pancreatitis. Results of embolization of pseudoaneurysms with coils showed high success rate and good short \& long term outcome. More research is required to search for better embolization materials which can minimize the risk of rebleeds or other complications.

Table 1. Characteristics of the patients enrolled in the study

\begin{tabular}{|l|l|}
\hline Variable & $\boldsymbol{N}=\mathbf{3 8}$ \\
\hline Males & 32 \\
\hline Age & Mean age 38.4 years \pm 4.32 years \\
\hline Patients with acute pancreatitis & 10 \\
\hline Patients with chronic pancreatitis & 28 \\
\hline Vascular complication & 33 \\
\hline Pseudoaneurysm & 5 \\
\hline Arterial wall erosion &
\end{tabular}

Table 2. Artery involved in pseudoaneurysm in patients with acute and chronic pancreatitis

\begin{tabular}{|c|c|}
\hline \multicolumn{2}{|c|}{ Site of pseudoaneurysm in acute pancreatitis $(n=10)$} \\
\hline Splenic artery & $4(40 \%)$ \\
\hline Gastroduodenal artery & $3(30 \%)$ \\
\hline Inferior pancreatico duodenal artery & $1(10 \%)$ \\
\hline Left gastric artery & $2(20 \%)$ \\
\hline \multicolumn{2}{|c|}{ Site of pseudoaneurysm in chronic pancreatitis $(n=28)$} \\
\hline Splenic artery & $11(39 \%)$ \\
\hline Gastroduodenal artery & $9(32 \%)$ \\
\hline Left gastroduodenal artery & $4(15 \%)$ \\
\hline Common hepatic artery & $2(7 \%)$ \\
\hline Inferior pancreatico duodenal artery & $2(7 \%)$ \\
\hline
\end{tabular}

Table 3. Method of embolization used in the patients

\begin{tabular}{|l|l|}
\hline Method used & $\boldsymbol{N}(\boldsymbol{\%})$ \\
\hline Coil only & $29(76 \%)$ \\
\hline Coil and glue & $7(18 \%)$ \\
\hline Coil and gelfoam & $2(6 \%)$ \\
\hline
\end{tabular}




\section{References}

[1]. Swaroop VS, Chari ST, Clain JE. Severe acute pancreatitis. JAMA 2004; 291:2865

[2]. Flati G, Andrén-Sandberg A, La Pinta M, Porowska B, Carboni M. Potentially fatal bleeding in acute pancreatitis: pathophysiology, prevention, and treatment. Pancreas. 2003:26:8-14

[3]. Stroud WH, Cullom JW, Anderson MC. Hemorrhagic complications of severe pancreatitis. Surgery 1981; 90:657-65

[4]. Edelman DA, Sugawa C. Lower gastrointestinal bleeding: a review. Surg Endosc 2007; 21:514.

[5]. Wee E. Management of nonvariceal upper gastrointestinal bleeding. J Postgrad Med 2011; 57:161.

[6]. Darcy M. Treatment of lower gastrointestinal bleeding: vasopressin infusion versus embolization. J Vasc Interv Radiol 2003; 14:535.

[7]. Ramaswamy RS, Choi HW, Mouser HC, et al. Role of interventional radiology in the management of acute gastrointestinal bleeding. World J Radiol 2014; 6:82

[8]. Yata S, Ihaya $\mathrm{T}$, Kaminou $\mathrm{T}$, et al. Transcatheter arterial embolization of acute arterial bleeding in the upper and lower gastrointestinal tract with N-butyl-2-cyanoacrylate. J Vasc Interv Radiol 2013; 24:422.

[9]. Toyoda H, Nakano S, Kumada T, et al. Estimation of usefulness of N-butyl-2-cyanoacrylate-lipiodol mixture in transcatheter arterial embolization for urgent control of life-threatening massive bleeding from gastric or duodenal ulcer. J Gastroenterol Hepatol 1996; 11:252.

[10]. Ripoll C, Bañares R, Beceiro I, et al. Comparison of transcatheter arterial embolization and surgery for treatment of bleeding peptic ulcer after endoscopic treatment failure. J Vasc Interv Radiol 2004; $15: 447$.

[11]. Loffroy R, Rao P, Ota S, et al. Embolization of acute nonvariceal upper gastrointestinal hemorrhage resistant to endoscopic treatment: results and predictors of recurrent bleeding. Cardiovasc Intervent Radiol 2010; 33:1088.

[12]. Lundgren JA, Matsushima K, Lynch FC, et al. Angiographic embolization of nonvariceal upper gastrointestinal bleeding: predictors of clinical failure. J Trauma 2011; 70:1208.

[13]. Funaki B. Endovascular intervention for the treatment of acute arterial gastrointestinal hemorrhage. Gastroenterol Clin North Am $2002 ; 31: 701$

[14]. Mirsadraee S, Tirukonda P, Nicholson A, et al. Embolization for non-variceal upper gastrointestinal tract haemorrhage: a sy 